\section{nephron}

Practice
Nephron 2017; 137:113-123

DOI: $10.1159 / 000477474$
Received: January 4, 2017

Accepted after revision: May 7, 2017

Published online: June 30, 2017

\title{
Empirically Reduced Dosages of Tinzaparin in Patients with Moderate-to-Severe Renal Insufficiency Lead to Inadequate Anti-Xa Levels
}

\author{
Renske H. Olie ${ }^{a, b} \quad$ Nathalie E.L. Meertens ${ }^{b} \quad$ Yvonne M.C. Henskens ${ }^{c}$ \\ Hugo ten Cate ${ }^{a, b, d}$ \\ ${ }^{a}$ Department of Internal Medicine, Maastricht University Medical Center+ (MUMC+), ${ }^{b}$ Thrombosis Expertise \\ Center, MUMC+, ${ }^{\mathrm{C} C e n t r a l}$ Diagnostic Laboratory, MUMC+, and ${ }^{\mathrm{d}}$ Laboratory for Clinical Thrombosis and \\ Hemostasis, Maastricht University, Maastricht, The Netherlands
}

\section{Keywords}

Low molecular weight heparin - Anti-Xa activity .

Anticoagulation therapy $\cdot$ Renal insufficiency . Dosage

adjustment

\begin{abstract}
Background: Due to the higher molecular weight of tinzaparin, the low molecular weight heparin (LMWH) is less dependent on renal excretion than other $\mathrm{LMWH}$ preparations. However, several international guidelines recommend the same preemptive dosage reduction for all therapeutic dose LMWHs prescribed in renal insufficient patients, to ensure that there is no accumulation of anticoagulant activity and increased risk of bleeding. This study is aimed at assessing whether a preemptive dosage reduction of tinzaparin in all renal insufficient patients (comprising 25\% reduction in patients with Modification of Diet in Renal Disease - estimated glomerular filtration rate (MDRD-eGFR) $30-60 \mathrm{~mL} /$ $\mathrm{min} / 1.73 \mathrm{~m}^{2}$ and $50 \%$ reduction in patients with MDRD-eGFR $<30 \mathrm{~mL} / \mathrm{min} / 1.73 \mathrm{~m}^{2}$ ) leads to adequate anti-Xa levels. Methods: We selected the anti-Xa levels of in-hospital patients ( $\geq 18$ years) with moderate-to-severe renal insufficiency (MDRD-eGFR $<60 \mathrm{~mL} / \mathrm{min} / 1.73 \mathrm{~m}^{2}$ ), on therapeutic dosages of tinzaparin. Anti-Xa levels were measured using a chromogenic assay. Results: Preemptive dosage reduction resulted
\end{abstract}

\section{KARGER}

(C) 2017 S. Karger AG, Basel

E-Mail karger@karger.com

www.karger.com/nef in a median anti-Xa activity of $0.50 \mathrm{IU} / \mathrm{mL}$ (interquartile range [IQR] 0.38-0.60). In $92.3 \%$ of patients the anti-Xa level was below the target anti-Xa level of $>0.85 \mathrm{IU} / \mathrm{mL}$ for therapeutic indications. Unadjusted dosages led to a median anti-Xa activity of $0.74 \mathrm{IU} / \mathrm{mL}$ (IQR 0.56-0.92). The preemptive dosage reduction was significantly associated with anti-Xa activity below therapeutic range $(p=0.007)$. No difference in anti-Xa activity was observed between patients with moderate $(0.71$ $\mathrm{IU} / \mathrm{mL}$, IQR $0.61-0.95)$ versus severe $(0.65 \mathrm{IU} / \mathrm{mL}$, IQR 0.41 1.06) renal insufficiency in whom an unadjusted dose had been administered $(p=0.77)$. None of the anti-Xa levels were above the upper margin of the presumed therapeutic range of $2.0 \mathrm{IU} / \mathrm{mL}$. Conclusion: In renal insufficient patients, the preemptive dosage reduction of tinzaparin leads to inadequate anti-Xa levels.

(c) 2017 S. Karger AG, Basel

\section{Introduction}

Low-molecular-weight-heparins (LMWHs) are anticoagulants formed by the depolymerization of unfractioned heparin (UFH) [1]. In the last decades, LMWHs have largely replaced UFH as anticoagulants, because LMWHs have been shown to be at least as efficient and safe and more convenient than UFH in many clinical sit-

Dr. Renske H. Olie

Department of Internal Medicine

Maastricht University Medical Center+

PO Box 5800, NL-6202 AZ Maastricht (The Netherlands)

E-Mail Renske.olie@ mumc.nl 
uations. Owing to their predictable anticoagulant effect, regular laboratory monitoring is not deemed generally necessary. However, anti-Xa activity measurements may be used to monitor the anticoagulant effect in certain situations in which the pharmacokinetics of LMWH is less well known, such as renal insufficiency, under- or overweight, pregnancy or childhood [2].

LMWHs are partially metabolized by depolymerization and/or desulfation and excreted preferentially via the kidneys, in contrast to UFH. Reduced renal clearance of LMWHs in patients with renal failure may lead to the accumulation of LMWH. As a result, the anticoagulant effects may be stronger. Whether a reduction in renal elimination will result in a clinically relevant accumulation of anticoagulant activity and therefore an increased risk of bleeding depends on several factors, including the severity of renal impairment, the dose administered, the duration of treatment and the type of LMWH used. The molecular weight of LMWHs may influence the balance between renal and non-renal elimination; the higher the molecular weight, the less important is the renal excretion for the overall elimination of the LMWH. The chains of higher molecular mass may bind to saturable sites of endothelial cells, reticulo-endothelial cells and mononuclear phagocytes, so that the cellular clearance is likely to be significant and renal clearance is less important for the elimination of the LMWH [3].

Tinzaparin is known to have the highest average molecular weight (approximately 6,500 Da) of all marketed LMWH preparations [3]. Several clinical studies indicate that there is no clinically significant increase in anti-Xa activity after repeated once-daily therapeutic doses of tinzaparin in patients with renal impairment [4-6]. However, the accumulation of LMWHs has been reported for other LMWH preparations, such as nadroparin and enoxaparin [7-10]. Currently, several international guidelines recommend dose reduction and/or anti-Xa monitoring if patients with moderate-to-severe renal insufficiency are treated with therapeutic dose of LMWH, to ensure that there is no accumulation of anticoagulant activity and increased risk of bleeding [2, 11]. Accordingly, in our hospital guideline we recommend a dose reduction of $25 \%$ for patients with moderate renal insufficiency (Modification of Diet in Renal Disease - estimated glomerular filtration rate [MDRD-eGFR] 30-60 mL/ $\mathrm{min} / 1.73 \mathrm{~m}^{2}$ ) and a dose reduction of $50 \%$ for patients with severe renal insufficiency (MDRD-eGFR $<30 \mathrm{~mL}$ / $\mathrm{min} / 1.73 \mathrm{~m}^{2}$ ) after a first full dose (100\%) has been administered. These dose reductions for LMWHs are in ac- cordance with the current guideline of the Dutch Federation of Nephrologists [12]. For patients on therapeutic LMWH for more than 3 days, monitoring of anti-Xa activity is recommended.

This study was aimed at assessing whether this preemptive dosage reduction of tinzaparin in patients with renal insufficiency still leads to adequate anti-Xa levels. To verify the appropriateness of this dosage reduction, we analyzed anti-Xa levels in renal insufficient patients who were monitored by anti-Xa activities, and retrospectively collected clinical data from the electronic medical records. Our main objective was to calculate the percentage of anti-Xa activities that fell within the therapeutic range. Here, we report these observational data.

\section{Subjects and Methods}

We present results of a retrospective cohort study on anti-Xa levels in hospitalized patients with renal insufficiency and anticoagulation with tinzaparin in whom a preemptive dosage reduction was applied.

\section{Patients}

This retrospective cohort study was conducted in a tertiary care hospital in The Netherlands (Maastricht University Medical Center+, Maastricht, The Netherlands) between July 2014 and July 2015 . All hospitalized patients $\geq 18$ years, with MDRD-eGFR $<60$ $\mathrm{mL} / \mathrm{min} / 1.73 \mathrm{~m}^{2}$, who were treated with therapeutic dosages of tinzaparin and monitored by anti-Xa levels, were included in our study. Patients admitted at the intensive care unit were not included, since in our hospital most intensive care unit patients were being treated with twice-daily nadroparin instead of once-daily tinzaparin. The serum creatinine value preceding the start of tinzaparin was used to calculate eGFR using the 4-variable MDRD formula [13]. For follow-up measurements of anti-Xa levels, whether or not after additional dosage adjustments, MDRD was once again calculated using the most recent serum creatinine value preceding the newer anti-Xa level measurement.

\section{Dosage Adjustments}

Our local clinical guideline dictates a preemptive dosage reduction of all therapeutic dosages of LMWHs for patients with renal failure, followed by an anti-Xa level measurement if LMWH is continued for more than 3 days. This guideline applies to all patients in our hospital with MDRD-eGFR $<60 \mathrm{~mL} / \mathrm{min} / 1.73 \mathrm{~m}^{2}$, regardless of the origin of renal failure, regardless of the reason for admission or ward and regardless of the indication for therapeutic LMWH. Tinzaparin is the most prescribed LMWH for therapeutic indications on most wards, with a weight-based dosing scheme (Table 1a). For this weight-based dose, we apply a preemptive dosage reduction of $25 \%$ for patients with moderate renal insufficiency (MDRD-eGFR 30-60 mL/min/1.73 $\mathrm{m}^{2}$ ) and a dosage reduction of $50 \%$ for patients with severe renal insufficiency (MDRD-eGFR $<30 \mathrm{~mL} / \mathrm{min} / 1.73 \mathrm{~m}^{2}$ ) after the first full dose (100\%) has been ad- 
Table 1.

a Dosing guidelines for tinzaparin once-daily

\begin{tabular}{ll}
\hline Bodyweight, kg & Dosage \\
\hline$<60$ & $0.5 \mathrm{~mL}(10,000 \mathrm{IE}$ anti-Xa) \\
$60-80$ & $0.7 \mathrm{~mL}(14,000 \mathrm{IE}$ anti-Xa) \\
$80-100$ & $0.9 \mathrm{~mL}(18,000 \mathrm{IE}$ anti-Xa) \\
$100-120$ & $1.0 \mathrm{~mL}(20,000 \mathrm{IE}$ anti-Xa $)$ \\
$120-140$ & $1.2 \mathrm{~mL}(24,000 \mathrm{IE}$ anti-Xa) \\
\hline
\end{tabular}

b Local guideline for dosage reduction in patients with renal insufficiency

\begin{tabular}{ll}
\hline Renal function & Dosage \\
\hline GFR 30-60 mL/min & Give a first full weight-based dose \\
& Continue LMWH with a 25\% dosage reduction \\
& Measure anti-Xa activity when continuing LMWH $>3$ days \\
& Consider dosage adjustment when anti-Xa levels are $<0.85$ or $>2.0 \mathrm{IU} / \mathrm{mL}$ \\
\hline GFR $<30 \mathrm{~mL} / \mathrm{min}$ & Give a first full weight-based dose \\
& Continue LMWH with a $50 \%$ dosage reduction \\
& Measure anti-Xa activity when continuing LMWH $>3$ days \\
& Consider dosage adjustment when anti-Xa levels are $<0.85$ or $>2.0 \mathrm{IU} / \mathrm{mL}$ \\
\hline
\end{tabular}

GFR, glomerular filtration rate; LMWH, low molecular weight heparin.

ministered (Table 1b). Prophylactic dosages were neither adjusted nor monitored by anti-Xa activity measurement and were not evaluated in this study.

\section{Anti-Xa Activity Measurements}

All anti-Xa levels in the period July 2014-July 2015 were retrieved. From this file, we selected the anti-Xa levels determined in hospital-admitted patients $\geq 18$ years with moderate-to-severe renal insufficiency (MDRD-eGFR $<60 \mathrm{~mL} / \mathrm{min} / 1.73 \mathrm{~m}^{2}$ ) on therapeutic dosages of tinzaparin. Anti-Xa activities were ordered by the treating physicians during the hospital stay. Anti-Xa levels were ideally measured $4 \mathrm{~h}$ after the administration of tinzaparin. The time of tinzaparin administration is automatically recorded in the electronic medical record, as is the time the blood sample arrives at the hospital central laboratory. Samples tend to arrive at the central laboratory within 15 min after the sample has been collected and thereby, the time interval between tinzaparin injection and the moment of anti-Xa measurement could be exactly calculated. Blood samples were collected in sodium citrate tubes $(3.2 \%$, $0.105 \mathrm{M}, \mathrm{BD}$ vacutainer, Plymouth, UK). Platelet-poor plasma was obtained by 2 centrifugation steps (room temperature): $5 \mathrm{~min}$ at $2,500 \mathrm{~g}$ followed by $10 \mathrm{~min}$ at $10,000 \mathrm{~g}$. Plasma samples were frozen immediately and subsequently anti-Xa activity was measured using a chromogenic technique on the same day, according to the instructions of the manufacturer (Biophen, Heparin test, Hyphen Biomed) on a CS2100i (Sysmex). The target range for peak anti-Xa levels (measured $4 \mathrm{~h}$ after dosing) with once-daily tinzaparin is $0.85-2.0$ units $/ \mathrm{mL}[2,14]$. If anti-Xa levels fell outside this range, it was at the treating physician's discretion to apply subsequent dosage adjustments and to order additional anti-Xa measurements. Data regarding treatment and outcome were retrospective- ly collected from the medical records. Because anti-Xa level measurement and subsequent dosage adjustments were part of the standard care in our hospital, neither institutional review board approval nor informed consent was required.

\section{Statistics}

Continuous variables are expressed as either mean \pm SD for normally distributed data or median with interquartile range otherwise. Categorical variables are expressed as counts and percentages. Anti-Xa activities were plotted against corresponding renal functions. We divided samples into three groups: group 1 - samples taken after dosage reduction according to the local guideline, group 2 - samples taken after unreduced dosages and group 3 - samples taken after dosages even lower than the local guideline recommended were administered. We calculated the proportions of anti-Xa levels that fell within the reference range, and Fisher's Exact test was used to test whether the proportion of anti-Xa levels within the therapeutic range varied across different groups of guideline adherence. Furthermore, samples after unreduced dosages and samples after dosage reduction were compared using non-parametric tests for continuous variables (Mann-Whitney U test or Kruskal-Wallis test). Follow-up measurements in patients on same dosage were compared using non-parametric tests for 2 paired samples (Wilcoxon signed rank test). Finally, we divided all samples into 4 subgroups (groups A-D) based on the administered dose as a percentage of standard tinzaparin dose of $175 \mathrm{IU} / \mathrm{kg}$. Mann-Whitney U test was used to test the differences between moderate and severe renal insufficiency in these dosage groups. Statistical analyses were performed with IBM SPSS statistics version 23.0. Statistical significance was assumed at $p<0.05$. 
Fig. 1. Inclusion of anti-Xa samples for analysis.

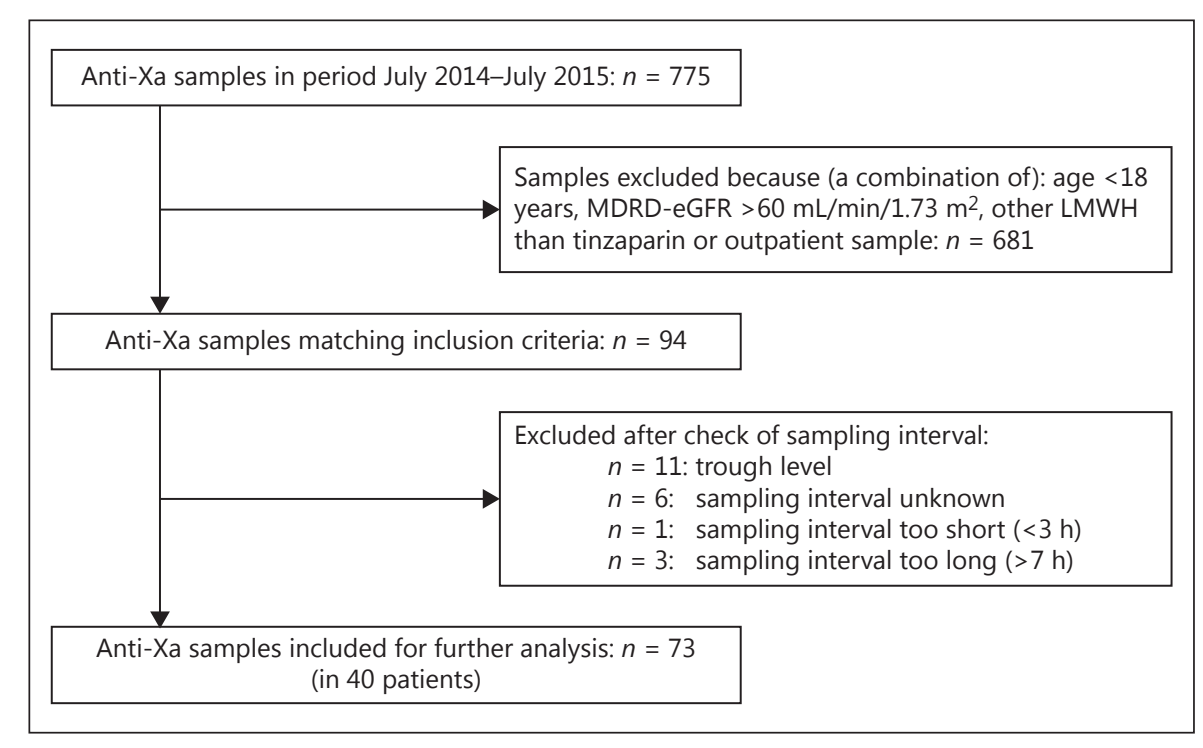

\section{Results}

A total of 775 anti-Xa levels in the period July 2014July 2015 were retrieved. After the selection of anti-Xa levels, determined in hospitalized patients $\geq 18$ years with renal insufficiency and on therapeutic dosages of tinzaparin, a total of 94 samples were identified. However, 21 samples had to be excluded from further analysis because they were trough levels $(n=11)$ or because the time interval between tinzaparin administration and blood sample withdrawal was either unknown $(n=6)$, too short $(<3 \mathrm{~h}$, $n=1)$, or too long $(>7 \mathrm{~h}, n=3)$. Thus, a total of 73 samples from 40 patients could be included for further analysis (Fig. 1). The median age of patients was 71 years (minmax: 26-91) and 35\% of included patients were males. Renal insufficiency was severe (MDRD-eGFR $<30 \mathrm{~mL}$ / $\left.\mathrm{min} / 1.73 \mathrm{~m}^{2}\right)$ in $55 \%$ of patients, and the mean renal clearance (MDRD-eGFR) was $30.3 \pm 12.8 \mathrm{~mL} / \mathrm{min} / 1.73 \mathrm{~m}^{2}$. First samples were taken after a median of 4 (min-max: 1-31) days after the start of tinzaparin. In 18 patients, one or more consecutive anti-Xa level measurements were performed. Patient and sample characteristics are shown in Table 2.

A total of 40 first samples and 33 consecutive samples was divided into 3 subgroups: samples taken after a priori dosage reduction according to the local guideline (group 1, $n=31$ ) and samples taken after an unadjusted dosage (group 2, $n=39$ ) or an over-adjusted dosage (group 3, $n=3$ ) had been administered. The median antiXa level was significantly different in these 3 groups, with highest anti-Xa levels in the unadjusted group and lowest anti-Xa levels in the over-adjusted group $(p<0.001)$.
Table 3 shows the median anti-Xa levels for different subgroups of patients with corresponding dosages of tinzaparin (in IU/kg). For analysis of median anti-Xa levels in these subgroups of patients, we excluded the group with over-adjusted dosages (group 3), because the group size was too small with only 3 samples to justify statistical analysis. Renal function and dosage regimens with corresponding anti-Xa levels of all 73 samples are shown in Figure 2.

For patients whose tinzaparin dosage was reduced according to the local guideline, the median anti-Xa activity of the first sample was 0.48 (interquartile range [IQR] $0.38-0.60) \mathrm{IU} / \mathrm{mL}$. In $92.3 \%$ of these samples, the anti-Xa level fell below the target range of $\geq 0.85 \mathrm{IU} / \mathrm{mL}$. The median anti-Xa activity was not different for patients with moderate versus severe renal insufficiency $(0.47$ [IQR $0.38-0.72] \mathrm{IU} / \mathrm{mL}$ vs. 0.50 [IQR $0.37-0.59] \mathrm{IU} / \mathrm{mL}, p=$ 0.68 ; Fig. 3 ). In daily practice, not all patients were treated according to this guideline; hence, they received unadjusted dosages of tinzaparin. The median anti-Xa activity of first samples after treatment with unadjusted tinzaparin dosages was 0.71 (IQR 0.55-0.95) IU/mL, which was significantly higher than median anti-Xa levels after reduced dosages were administered ( $p=0.019)$. But still, in $69.2 \%$ of these samples anti-Xa levels were below the target range of $>0.85 \mathrm{IU} / \mathrm{mL}$, compared to $92.3 \%$ in the a priori reduced group. Fisher's exact test indicated a significant association between guideline dosage adherence and anti-Xa levels falling below the target range ( $p=$ $0.007)$. The median anti-Xa level after unadjusted dosages was not different for groups with moderate versus severe renal insufficiency ( $p=0.77$; Fig. 3$)$. 
Table 2. Baseline characteristics

\begin{tabular}{|c|c|}
\hline \multicolumn{2}{|l|}{ Patient characteristics $(n=40)$} \\
\hline Age, years, median (min-max) & $71(26-91)$ \\
\hline Gender, male, $n(\%)$ & $14(35.0)$ \\
\hline Body weight, kg, mean \pm SD & $73.4 \pm 15.8$ \\
\hline $\begin{array}{l}\text { Days on tinzaparin to first anti-Xa level, } \\
\text { median (min-max) }\end{array}$ & $4.0(1-31)$ \\
\hline MDRD-eGFR, $\mathrm{mL} / \mathrm{min} / 1.73 \mathrm{~m}^{2}$, mean $\pm \mathrm{SD}$ & $30.3 \pm 12.8$ \\
\hline $50-59$ & $4(10.0)$ \\
\hline $40-49$ & $7(17.5)$ \\
\hline $30-39$ & $7(17.5)$ \\
\hline $20-29$ & $13(32.5)$ \\
\hline $10-19$ & $8(20.0)$ \\
\hline$<10$ & $1(2.5)$ \\
\hline \multicolumn{2}{|l|}{ Renal function on day $1, n(\%)$} \\
\hline $\begin{array}{l}\text { Moderate, MDRD-eGFR 30-60 } \\
\mathrm{mL} / \mathrm{min} / 1.73 \mathrm{~m}^{2}\end{array}$ & $18(45.0)$ \\
\hline Severe, MDRD-eGFR $<30 \mathrm{~mL} / \mathrm{min} / 1.73 \mathrm{~m}^{2}$ & $22(55.0)$ \\
\hline Renal replacement therapy & $0(0.0)$ \\
\hline \multicolumn{2}{|l|}{ Indication for anticoagulation, $n(\%)$} \\
\hline Atrial fibrillation & $18(45.0)$ \\
\hline Venous thromboembolism & $11(27.5)$ \\
\hline Mechanical valves & $1(2.5)$ \\
\hline Other & $10(25.0)$ \\
\hline \multicolumn{2}{|l|}{ Ward } \\
\hline Surgical & $11(27.5)$ \\
\hline Medical & $29(72.5)$ \\
\hline
\end{tabular}

Sample characteristics $(n=73)$

Sample, $n(\%)$

First sample

$40(54.8)$

Consecutive sample

$33(45.2)$

Interval between dosage and sample, $n(\%)$

Interval 3-5 h

$59(80.8)$

Interval 5-7 h

$14(19.2)$

Protocol adherence, $n(\%)$

Dosage according to protocol

Unadjusted (dosage above guideline)

$31(42.5)$

$39(53.4)$

$3(4.1)$

Values are expressed as mean with SDs or as median with minimum and maximum, based on the normal distribution for the data. Categorical data are expressed as $n(\%)$.

MDRD-eGFR, Modification of Diet in Renal Disease - estimated glomerular filtration rate.

\section{Follow-Up Therapy and Anti-Xa Measurement}

When anti-Xa levels fall below the therapeutic range of $\geq 0.85 \mathrm{IU} / \mathrm{mL}$, our guideline recommends dosage adjustment and follow-up measurement of anti-Xa level. In 18 patients, therapy was indeed changed based on anti-
Xa measurement; in 17 patients the dosage was increased and in 1 patient LMWH treatment was replaced by UFH (online suppl. Fig. 1, see www.karger.com/doi/ $10.1159 / 000477474)$. There were no patients in who the dosage had to be decreased based on anti-Xa level measurement. In patients whose dosage had to be increased to achieve anti-Xa levels within the therapeutic range, the median tinzaparin dosage needed was 225 (IQR 164255) IU/kg. A total of 33 consecutive samples in 18 patients were available for further analysis. Due to dosage adjustments in some of these patients, the median antiXa level in consecutive samples was significantly higher than in first samples (0.73 [IQR 0.55-0.95] vs. 0.55 [IQR $0.38-0.70], p=0.008$ ). Comparable to findings in first samples, we found no signs of accumulation after unreduced tinzaparin dosages had been administered, as median anti-Xa activity in patients with severe renal insufficiency was $0.63 \mathrm{IU} / \mathrm{mL}$ (IQR 0.52-0.84) compared to $0.88 \mathrm{IU} / \mathrm{mL}$ (IQR $0.67-0.96$ ) in patients with only moderate renal insufficiency ( $p=0.09$; Table 3$)$. In 14 out of 17 patients in whom dosage had been increased based on inadequate anti-Xa levels in the first sample, one or more consecutive samples were available. The median anti-Xa level of these samples was $0.85 \mathrm{IU} / \mathrm{mL}$, proving that dosage adjustment led to anti-Xa levels within the therapeutic range.

In 7 patients, a second anti-Xa level measurement was done while on the same tinzaparin dosage after a median time interval of 8 (min-max: 2-10) days. However, we found no signs of accumulation, as the median anti-Xa activity of this second measurement on the same dosage did not increase compared to the first anti-Xa sample $(p=0.398$; Fig. 4).

\section{Moderate versus Severe Renal Insufficiency}

To explore whether the effect of dosage reduction was different between patients with severe and moderate renal insufficiency, we divided all samples, both first and consecutive measurements, into 4 dosage groups according to the percentage of standard tinzaparin dosage of $175 \mathrm{IU} / \mathrm{kg}$. To achieve an anti-Xa activity within a therapeutic range of $\geq 0.85 \mathrm{IU} / \mathrm{mL}$, in some patients tinzaparin dosage had to be increased even higher than the standard dosage of $175 \mathrm{IU} /$ $\mathrm{kg}$. We divided the samples into 4 subgroups: group A: $>110 \%$ of standard dosage $(n=20)$; group B: $90-110 \%$ of standard dosage $(n=17)$; group C: $65-90 \%$ of standard dosage $(n=19)$ and group D: $<65 \%$ of standard dosage $(n=$ 17). Within all these 4 groups, anti-Xa levels did not differ significantly between samples taken in patients with severe or moderate renal insufficiency (Table 4). 
Fig. 2. Anti-Xa levels with corresponding renal function and dosage regimens. Each symbol represents an anti-Xa level measurement. Blue dots represent an anti-Xa level measurement in patients who received tinzaparin dosages adjusted according to the guideline $(n=31)$. Red triangles represent anti-Xa level measurements in patients who received unadjusted tinzaparin dosages $(n=39)$. Green triangles represent anti-Xa level measurements in patients who received tinzaparin dosages lower than the guideline $(n=3)$. Dashed lines represent the upper and lower limit of the therapeutic range $(0.85-2.0 \mathrm{IU} / \mathrm{mL})$. MDRD-eGFR, Modification of Diet in Renal Disease - estimated glomerular filtration rate.

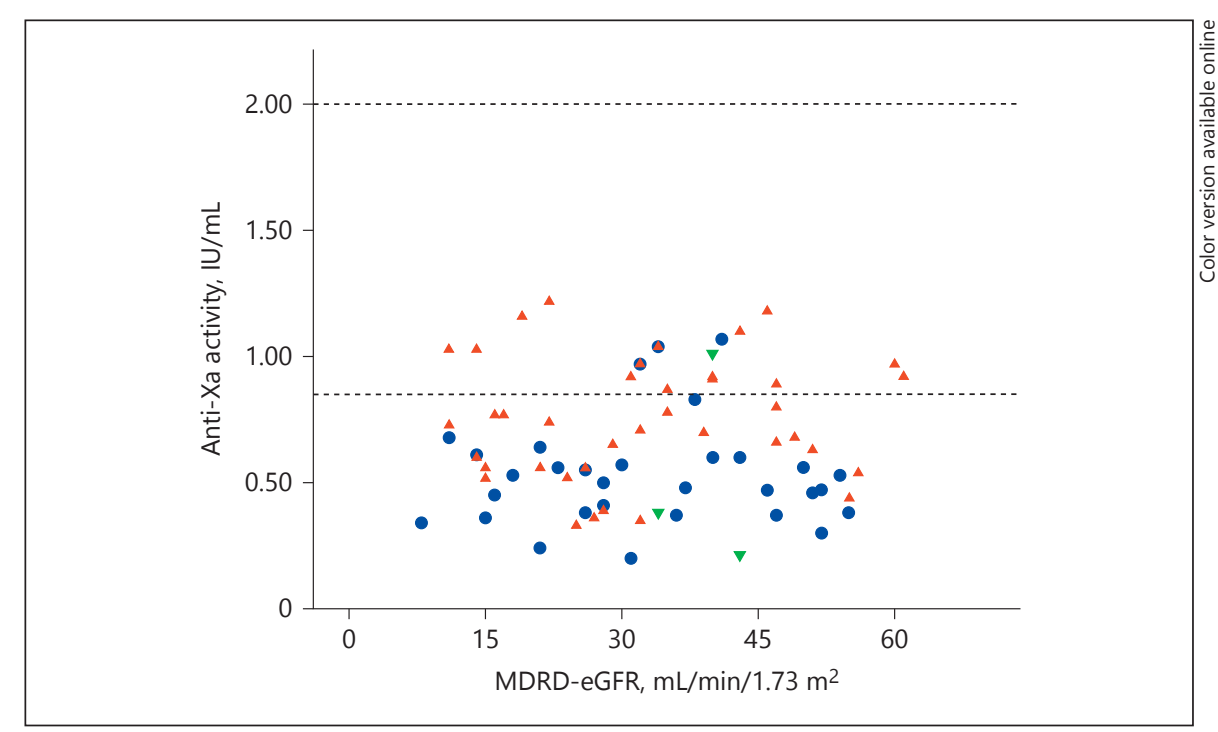

Table 3. Results: median anti-Xa levels (in IU/mL) and median tinzaparin dosage (in IU/kg) in 3 subgroups; group 1( $n=31$ ) - samples taken after a priori dosage reduction of tinzaparin according to the local guideline, group $2(n=39)$ samples taken after unadjusted tinzaparin dosages and group $3(n=3)$ samples taken after overadjusted tinzaparin dosages had been administered

\begin{tabular}{|c|c|c|c|c|c|}
\hline & All $(n=73)$ & $\begin{array}{l}\text { According to protocol } \\
\text { group } 1(n=31)\end{array}$ & $\begin{array}{l}\text { Unadjusted } \\
\text { group } 2(n=39)\end{array}$ & $\begin{array}{l}\text { Overadjusted } \\
\text { group } 3(n=3)\end{array}$ & $p$ value \\
\hline \multicolumn{6}{|l|}{ Anti-Xa level measurements } \\
\hline \multicolumn{6}{|l|}{ Anti-Xa level, IU/mL, median (IQR) } \\
\hline First sample $\left(n=39^{*}\right)$ & $0.55(0.38-0.70)$ & $0.48(0.38-0.60)$ & $0.71(0.55-0.95)$ & & 0.019 \\
\hline \multicolumn{6}{|l|}{ Renal insufficiency } \\
\hline Severe $(n=21)$ & & $0.50(0.37-0.59)$ & $0.65(0.41-1.06)$ & & 0.103 \\
\hline Consecutive sample $\left(n=31^{*}\right)$ & $0.73(0.55-0.95)$ & $0.56(0.42-0.79)$ & $0.78(0.59-0.93)$ & & 0.139 \\
\hline \multicolumn{6}{|l|}{ Renal insufficiency } \\
\hline Moderate $(n=21)$ & & $0.56(0.42-0.79)$ & $0.88(0.67-0.96)$ & & 0.076 \\
\hline Severe $(n=10)$ & & - & $0.63(0.52-0.84)$ & & - \\
\hline \multicolumn{6}{|l|}{ Dosage tinzaparin, IU/kg } \\
\hline Severe $(n=31)$ & & $100(91-109)$ & $165(149-196)$ & & $<0.001$ \\
\hline \multicolumn{6}{|l|}{ Dosage, IU/kg, median (IQR) } \\
\hline \multicolumn{6}{|l|}{ First sample $\left(n=39^{*}\right)$} \\
\hline Moderate & & $140(125-153)$ & $206(186-231)$ & & 0.001 \\
\hline Severe & & $100(91-109)$ & $173(153-190)$ & & $<0.001$ \\
\hline \multicolumn{6}{|l|}{ Consecutive samples $\left(n=31^{*}\right)$} \\
\hline Moderate & & $156(117-175)$ & $236(212-255)$ & & 0.007 \\
\hline Severe & & - & $165(139-308)$ & & - \\
\hline $\begin{array}{l}\text { Values are expressed as median w } \\
\text { \# Mann-Whitney test to compare } \\
\text { ^ IQR not calculable due to the sn } \\
\text { * Group } 3 \text { samples were excluded }\end{array}$ & $\begin{array}{l}\text { interquartile rang } \\
\text { oups } 1 \text { and } 2 \text { and } \mathrm{K} \\
11 \text { sample size. } \\
\mathrm{r} \text { further analysis, } \mathrm{b}\end{array}$ & $\begin{array}{l}\text { (IQR). } \\
\text { uskal-Wallis test to comp } \\
\text { cause the group size was }\end{array}$ & $\begin{array}{l}\text { are all the } 3 \text { groups. } \\
\text { oo small }(n=3) \text { to }\end{array}$ & if & ysis. \\
\hline
\end{tabular}


Fig. 3. Anti-Xa activity after administration of reduced tinzaparin dosage according to the local protocol $(n=31)$ or after unreduced dosages of tinzaparin $(n=39)$ in patients with moderate and severe renal insufficiency.
Fig. 4. Follow-up measurements within patients on same tinzaparin dosage. In 7 patients, a second anti-Xa level was measured while on the same tinzaparin dosage. This second anti-Xa level was not statistically different from the first anti-Xa level measurement in these patients $(p=0.398)$.
Dosage tinzaparin administered
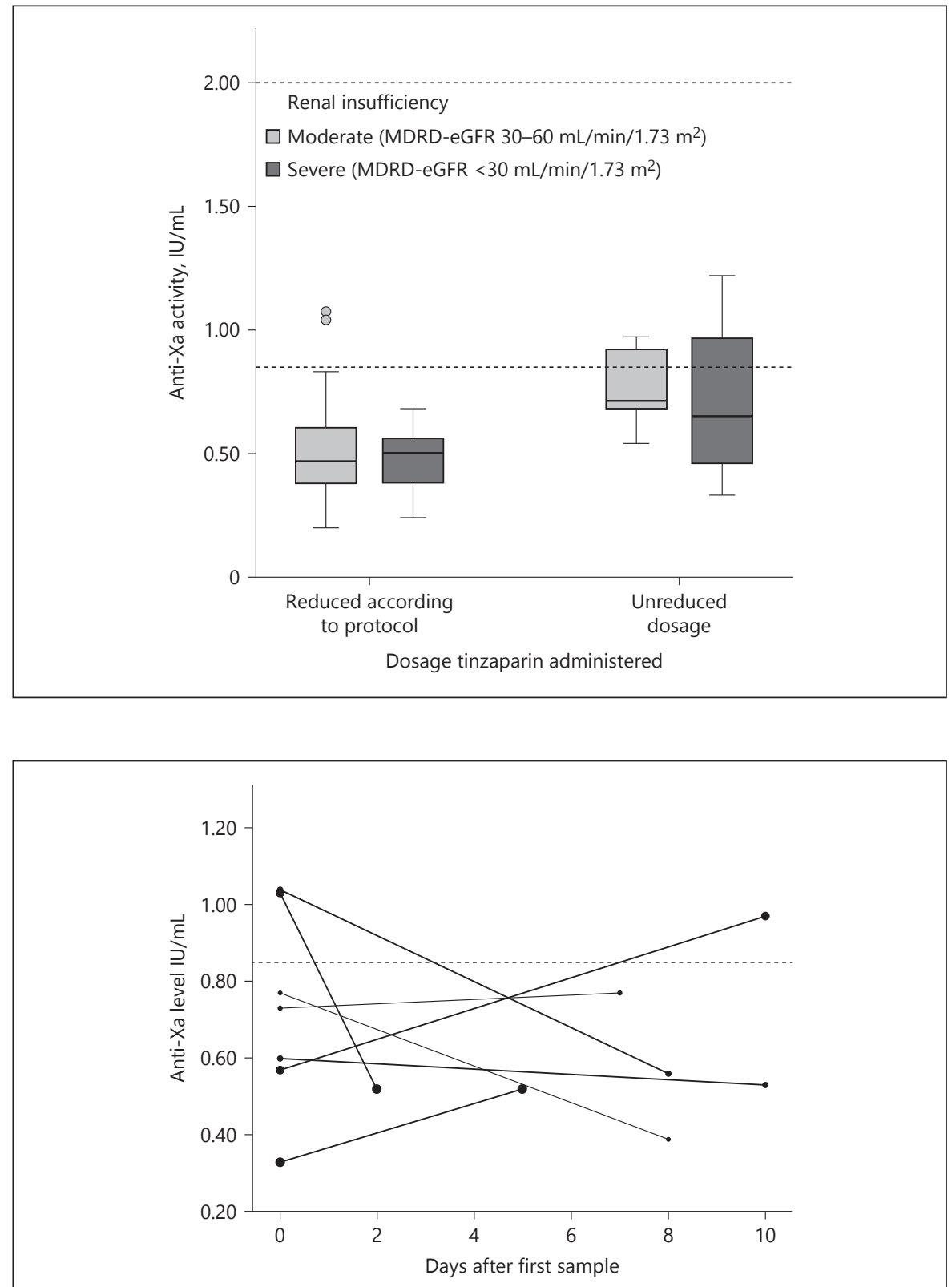

Table 4. Anti-Xa level, IU/mL per dosage category for moderate versus severe renal insufficiency

\begin{tabular}{|c|c|c|c|c|c|c|}
\hline & $\begin{array}{l}\text { Dosing group (percent } \\
\text { age of standard dosage } \\
\text { of } 175 \mathrm{IU} / \mathrm{kg} \text { ), } \%\end{array}$ & $\begin{array}{l}\text { Moderate renal } \\
\text { Insufficiency } \\
(n=42)\end{array}$ & $\begin{array}{l}\text { Patients in } \\
\text { subgroup, } n\end{array}$ & $\begin{array}{l}\text { Severe renal } \\
\text { Insufficiency } \\
(n=31)\end{array}$ & $\begin{array}{l}\text { Patients in } \\
\text { subgroup, } n\end{array}$ & $p$ value \\
\hline Group A $(n=20)$ & $>110$ & $0.90(0.70-0.97)$ & 16 & $0.82(0.54-1.17)$ & 4 & 0.93 \\
\hline Group B $(n=17)$ & $90-110$ & $0.56(0.49-0.75)$ & 9 & $0.74(0.40-0.77)$ & 8 & 0.77 \\
\hline Group C $(n=19)$ & $65-90$ & $0.47(0.37-0.77)$ & 12 & $0.56(0.45-0.65)$ & 7 & 0.45 \\
\hline Group D $(n=17)$ & $<65$ & $0.57(0.30-0.99)$ & 5 & $0.52(0.37-0.60)$ & 12 & 0.49 \\
\hline
\end{tabular}

Values are expressed as median with interquartile ranges.

$p$ values were calculated using the Mann-Whitney test.

Empirically Reduced Dosages of Tinzaparin in Patients
Nephron 2017;137:113-123 DOI: $10.1159 / 000477474$ 


\section{Complications}

None of the anti-Xa levels were above the upper margin of the presumed therapeutic range of $2.0 \mathrm{IU} / \mathrm{mL}$. Seven patients experienced a bleeding event during hospital stay; 3 cases of gastrointestinal bleeding, 2 cases of severe hematomas, 1 case of hematuria and 1 patient had postoperative bleeding due to a complication of the procedure. The median MDRD-eGFR of patients with a bleeding complication was 23.0 (IQR $20-32$ ) $\mathrm{mL} / \mathrm{min} / 1.73 \mathrm{~m}^{2}$, but did not differ significantly from patients without bleeding complication (MDRD-eGFR 28.0 [IQR 21-41] $\left.\mathrm{mL} / \mathrm{min} / 1.73 \mathrm{~m}^{2}, p=0.244\right)$. The median anti-Xa level of patients with a bleeding complication was 0.56 (IQR 0.34-0.64) IU/mL, none of them had an anti-Xa level $>1.0 \mathrm{IU} / \mathrm{mL}$. Numbers are too small to evaluate the association between anti-Xa activity and the occurrence of bleeding.

Four patients experienced a thromboembolic event during treatment with tinzaparin; 2 acute peripheral arterial occlusions, 1 myocardial infarction and 1 venous thromboembolism. The median anti-Xa level of patients with a thrombotic event was 0.59 (IQR 0.30-0.96) IU/mL. Numbers are too small to evaluate the association between anti-Xa activity and the occurrence of thrombotic events.

\section{Discussion}

This study was aimed at evaluating the standard dosage reduction of tinzaparin for all patients with renal impairment, as is currently proposed in our hospital guideline based on recommendations in several international guidelines $[2,11,12]$. Our main finding is that an a priori dosage reduction of 25 or $50 \%$ for patients with moderate or severe renal insufficiency respectively, leads to adequate anti-Xa levels in only $7.7 \%$ of patients. Subsequent dosage adjustments were necessary to realize an anti-Xa level of $\geq 0.85 \mathrm{IU} / \mathrm{mL}$. No patients had anti-Xa levels $>2.0$ $\mathrm{IU} / \mathrm{mL}$, a threshold proposed as the upper limit of the therapeutic range [2], neither patients with adjusted dosages, nor patients with renal insufficiency on full tinzaparin dose of $175 \mathrm{IU} / \mathrm{kg}$. Dosage reduction was significantly associated with anti-Xa falling out of therapeutic range, compared to a group of patients in whom unadjusted dosages had been administered. As far as we know, this is the first clinical study for evaluating a preemptive dosage reduction of tinzaparin in renal insufficient patients.

For tinzaparin, no prospective studies are available that include clinical outcomes in renal insufficient pa- tients evaluating different dosing strategies. The Innohep in Renal Insufficiency Study was stopped in 2008, because of an interim finding of an increase in all-cause mortality in patients who received standard dose tinzaparin compared to UFH [15]. The observed mortality difference could not be explained on the basis of bleeding or recurrent venous thromboembolism, and might have reflected an imbalance of risk factors at baseline. However, our results are in line with findings of other clinical studies [4-6], which showed no accumulation with unreduced tinzaparin dose, measured by anti-Xa activity, in elderly patients including renal insufficient patients. Siguret et al. [6] reported no increase in mean anti-Xa levels over a 10-day treatment period in 30 patients aged $>70$ years with a mean creatinine clearance of $40.6 \pm 15.3 \mathrm{~mL} / \mathrm{min}$ who were treated with full dose tinzaparin $(175 \mathrm{IU} / \mathrm{kg})$ once daily. No correlation was found between creatinine clearance and anti-Xa activity, as anti-Xa levels in patients with creatinine clearance between 20 and $30 \mathrm{~mL} / \mathrm{min}$ were comparable to anti-Xa levels in patients with creatinine clearance $>50 \mathrm{~mL} / \mathrm{min}$. Pautas et al. [4] showed that there was no accumulation of anti-Xa levels in 200 patients aged $>70$ years with a mean creatinine clearance of $51.2 \pm 22.9 \mathrm{~mL} / \mathrm{min}$, who were treated with standard weight-based dosages of tinzaparin. In a sub-study of the Innohep in Renal Insufficiency Study, 87 patients with a mean creatinine clearance of $40.8 \mathrm{~mL} / \mathrm{min}$ were included [5]. With an unreduced tinzaparin dosage, mean peak anti-Xa levels were $0.86 \mathrm{IU} / \mathrm{mL}$ on day $2 / 3$ and $0.87 \mathrm{IU} / \mathrm{mL}$ on day 5 , suggesting no significant accumulation. On day 5 of treatment, no statistically significant difference was observed between the mean anti-Xa levels in patients with moderate renal insufficiency and those with severe renal insufficiency: 0.84 vs. $0.96 \mathrm{IU} / \mathrm{mL}(p=0.125)$, respectively. Based on these studies, product information of tinzaparin suggests that a full therapeutic dose can be used for patients with creatinine clearance $>30 \mathrm{~mL} / \mathrm{min}$, while tinzaparin should be used cautiously when required in patients with creatinine clearance levels of $20-30 \mathrm{~mL} /$ min, as the optimal dosage in this population has not been established. According to the product information of tinzaparin, for the latter group of patients, tinzaparin should be initiated with anti-Xa monitoring and subsequent dosage adjustment, if the benefit outweighs the risk [16].

Our study focused on the use of tinzaparin in renal insufficient patients, but these results cannot simply be extrapolated to other LMWHs. Since the LMWHs differ in pharmacodynamic and pharmacokinetic profiles, ac- 
cumulation, as measured by anti-Xa activity, can occur with other LMWHs. In a study by Russcher et al. [17], this was shown for therapeutic dosages of nadroparin in renal insufficient patients. In patients with a MDRDeGFR $<60 \mathrm{~mL} / \mathrm{min} / 1.73 \mathrm{~m}^{2}$ an unadjusted nadroparin dosage led to anti-Xa levels higher than the therapeutic values in $60 \%$ of cases, implicating possible accumulation of LMWH in these patients. On the contrary, when an a priori dosage reduction of $25 \%$ was applied, the anti-Xa activity was adequate in $51 \%$ of measurements with only $19 \%$ of measurements being higher than the therapeutic range. Most evidence on altered pharmacokinetics in patients with renal insufficiency on LMWH exists for the use of enoxaparin [18]. When a therapeutic full dose of enoxaparin was administered, anti-Xa levels were significantly higher among patients with severe renal insufficiency (creatinine clearance $<30 \mathrm{~mL} / \mathrm{min}$ ) than those without $[7-9,19]$. A meta-analysis of therapeuticdose enoxaparin studies showed that major bleeding was significantly higher among patients with severe renal insufficiency compared to patients with better renal function (OR 3.88, 95\% CI 1.78-8.45). When the same analysis was done for 3 adjusted-dose enoxaparin studies, OR for major bleeding was lower, but with broad CIs (OR $0.58,95 \%$ CI $0.09-3.78$ ) [7]. The accumulation of enoxaparin in renal insufficient patients can be explained by the lower molecular weight (approximately 4,400 Da) of enoxaparin; the lower the molecular weight, the more important is the renal clearance for the overall elimination of LMWH [18]. For enoxaparin, clear recommendations for dosage reduction exist in several guidelines [2]. This difference in occurrence of accumulation for specific LMWHs was also illustrated in the review of Atiq et al. [20], who focused on the accumulation of prophylactic dosages of different LMWHs in patients with renal insufficiency.

Although the rationale for our local guideline of dosage reduction was fear of increased bleeding risk due to the accumulation of $\mathrm{LMWH}$, we might on the contrary have introduced the risk of therapy failure. A standard dose reduction by $50 \%$ in patients with severe renal insufficiency may even under-dose LMWH, as has previously been shown for enoxaparin [21, 22]. For enoxaparin, there is evidence that under-dosage may lead to loss of efficacy with a worse clinical outcome [23]. Following our results of inadequate anti-Xa levels after standard dosage reduction, we propose that in renal insufficient patients tinzaparin should not be routinely reduced in dose, but be monitored with anti-Xa activity, and the dose adjusted accordingly.

Empirically Reduced Dosages of

Tinzaparin in Patients
Anti-Xa Measurement and Therapeutic Range

What is the optimal anti-Xa level and what is it based on? Several guidelines suggest a therapeutic range for all once-daily therapeutic LMWHs of 1.0-2.0, but for tinzaparin a threshold of $\geq 0.85$ would be more convenient $[2$, 12]. Studies conducted in healthy individuals receiving tinzaparin showed that the mean anti-Xa peak level was approximately $0.8 \mathrm{IU} / \mathrm{mL}$ [24-27]. In these studies, $90 \%$ of patients had an anti-Xa peak level between 0.5 and 1.5 $\mathrm{IU} / \mathrm{mL}$ due to intersubject variability. Moreover, it should be noted that when a Gaussian distribution is assumed, with a mean anti-Xa level of $0.8 \mathrm{IU} / \mathrm{mL}$ in healthy volunteers, still 50\% will have therapeutic anti-Xa levels below this threshold. In the study of Siguret et al. [6], a mean anti-Xa level of $0.65 \mathrm{IU} / \mathrm{mL}( \pm 0.19)$ was found on day 5 of treatment in 30 elderly patients with a mean creatinine clearance of $40.8 \mathrm{~mL} / \mathrm{min}$, who were treated with full therapeutic dosages of tinzaparin. These results are in line with our median anti-Xa level of $0.74 \mathrm{IU} / \mathrm{mL}$ (IQR 0.560.92) in patients with unadjusted dosages and show that even with an unreduced dosage the threshold of $\geq 0.85 \mathrm{IU} / \mathrm{mL}$ could not always be reached.

It should be noted that although measuring anti-Xa levels is currently the generally accepted method to verify adequate LMWH dosing, there is discussion as to how it may be correlated to clinical events such as bleeding and thromboembolic complications [18]. Furthermore, whether the measurement of anti-Xa levels in this specific population of renal insufficient patients is appropriate for detecting patients at risk of bleeding or recurrent thromboembolic events is controversial $[28,29]$. In large cohorts of patients with acute coronary syndrome receiving enoxaparin, excessive peak anti-Xa levels have been shown to be associated with an increased risk of bleeding [23]. On the contrary, under-dosage of LMWH has been shown to lead to a loss of efficacy with a worse clinical outcome [23]. However, the threshold below which clinicians should be concerned about the risk of accumulation or under-dosage may vary from one LMWH to another [30]. In addition, the anti-Xa levels may not be fully representative of the antithrombotic properties of LMWH, since these agents have other effects as well, such as on platelet function and on tissue factor pathway inhibitor release from endothelium [2]. In fact, anti-Xa activity is a pharmacodynamic effect that is being used as a pharmacokinetic parameter [18]. However, as standard coagulation tests such as activated partial thromboplastin time cannot be used for LMWH monitoring, this surrogate parameter is the best available for clinical routine. One could argue that for the purpose of ruling out the possibil-

Nephron 2017;137:113-123

DOI: $10.1159 / 000477474$
121 
ity of accumulation, monitoring should be performed just before the application of the next dose, by measuring a trough level instead of a peak level.

Our study has several strengths. It is the first clinical report that evaluated standard dosage reduction of tinzaparin for all patients with renal impairment. It evaluates standard care in a tertiary care hospital, with all the difficulties in daily practice that come with it. Because we could only analyze renal insufficient patients who were monitored by anti-Xa measurements, as is required for all renal insufficient patients according to our hospital guideline, we have potentially missed patients in whom the treating physician did not follow the guideline. Due to the retrospective character of this study, bias in relation to prescribing strategies, dosage adjustments or number of anti-Xa measurements can clearly not be ruled out. Hence, we should be careful in generalizing the results of our study to the group of chronic kidney disease patients as a whole. Another important limitation of this study is that the objective of our analysis was to evaluate the impact of the preemptive dosage reduction as proposed in our guideline, and that it was not designed nor powered to correlate the resulting anti-Xa activities to clinical outcome. Adverse events such as thrombotic events or bleeding were not structurally recorded, but retrospectively derived from the electronic medical records. This might have led to an underestimation of the total number of adverse events, both bleeding and re-thrombotic. Prospective studies with clinical outcomes are needed, exploring the role of anti-Xa measurement in dose titration, to guide in optimal dosing of tinzaparin in renal insufficient patients.

\section{Conclusion}

Preemptive dosage reduction of tinzaparin in all patients with moderate-to-severe renal insufficiency leads to sub-therapeutic anti-Xa levels. Although the rationale of this dosage reduction was fear of increased bleeding risk due to accumulation of LMWH, we might on the contrary have introduced the risk of therapy failure. We argue that in renal insufficient patients tinzaparin should not be routinely reduced in dose, but started at the regular weight-based dose, and subsequently monitored with anti-Xa activity and the dose adjusted accordingly.

\section{Statement of Ethics}

This study did not require informed consent nor review/approval by the appropriate ethics committee.

\section{Disclosure Statement}

The authors have no conflicts of interest to declare.

\section{References}

1 Hirsh J, Levine MN: Low molecular weight heparin. Blood 1992;79:1-17.

2 Garcia DA, Baglin TP, Weitz JI, Samama MM: Parenteral anticoagulants: antithrombotic therapy and prevention of thrombosis, 9th ed: American college of chest physicians evidence-based clinical practice guidelines. Chest 2012;141:e24S-e43S.

3 Johansen KB, Balchen T: Tinzaparin and other low-molecular-weight heparins: what is the evidence for differential dependence on renal clearance? Exp Hematol Oncol 2013;2:21.

4 Pautas E, Gouin I, Bellot O, Andreux JP, Siguret V: Safety profile of tinzaparin administered once daily at a standard curative dose in two hundred very elderly patients. Drug Saf 2002;25:725-733.

5 Siguret V, Gouin-Thibault I, Pautas E, Leizorovicz A: No accumulation of the peak antifactor Xa activity of tinzaparin in elderly patients with moderate-to-severe renal impairment: the IRIS substudy. J Thromb Haemost 2011;9:1966-1972.

6 Siguret V, Pautas E, Fevrier M, Wipff C, Durand-Gasselin B, Laurent $\mathrm{M}$, Andreux JP,
d'Urso M, Gaussem P: Elderly patients treated with tinzaparin (Innohep) administered once daily (175 anti-Xa IU/kg): anti-Xa and antiIIa activities over 10 days. Thromb Haemost 2000;84:800-804.

7 Lim W, Dentali F, Eikelboom JW, Crowther MA: Meta-analysis: low-molecular-weight heparin and bleeding in patients with severe renal insufficiency. Ann Intern Med 2006; 144:673-684.

8 Chow SL, Zammit K, West K, Dannenhoffer $\mathrm{M}$, Lopez-Candales A: Correlation of antifactor Xa concentrations with renal function in patients on enoxaparin. J Clin Pharmacol 2003;43:586-590.

9 Becker RC, Spencer FA, Gibson M, Rush JE, Sanderink G, Murphy SA, Ball SP, Antman EM; TIMI 11A Investigators: Influence of patient characteristics and renal function on factor Xa inhibition pharmacokinetics and pharmacodynamics after enoxaparin administration in non-ST-segment elevation acute coronary syndromes. Am Heart J 2002;143:753-759.

10 Mismetti P, Laporte-Simitsidis S, Navarro C, Sie P, d'Azemar P, Necciari J, Duret JP, Gaud
C, Decousus H, Boneu B: Aging and venous thromboembolism influence the pharmacodynamics of the anti-factor $\mathrm{Xa}$ and antithrombin activities of a low molecular weight heparin (nadroparin). Thromb Haemost 1998;79:1162-1165.

11 KDIGO 2012 clinical practice guideline for the evaluation and management of chronic kidney disease. Kidney Int Suppl 2013;3:1150.

12 Nederlandse Federatie Voor Nefrologie, 2012. https://www.Nefro.Nl/richtlijnen/antistollingmet-lmwh-bij-nierinsuffici\%c3\%abntie-2012.

13 Levey AS, Coresh J, Greene T, Marsh J, Stevens LA, Kusek JW, Van Lente F; Chronic Kidney Disease Epidemiology Collaboration: Expressing the modification of diet in renal disease study equation for estimating glomerular filtration rate with standardized serum creatinine values. Clin Chem 2007;53:766772 .

14 Boneu B, de Moerloose P: How and when to monitor a patient treated with low molecular weight heparin. Semin Thromb Hemost 2001; 27:519-522. 
15 Leizorovicz A, Siguret V, Mottier D; Innohep ${ }^{\circledR}$ in Renal Insufficiency Study Steering Committee, Leizorovicz A, Siguret V, Mottier $\mathrm{D}$, Clonier F, Janas M, Stinson J, Townshend G, Maddalena M: Safety profile of tinzaparin versus subcutaneous unfractionated heparin in elderly patients with impaired renal function treated for acute deep vein thrombosis: the innohep ${ }^{\circledR}$ in renal insufficiency study (IRIS). Thromb Res 2011;128:27-34.

16 Innohep ${ }^{\circledR} 20,000 \mathrm{IU} / \mathrm{ml}$ Summary of Product Characteristics, 2016. http://www.Medicines. Org.Uk/emc/medicine/29740.

17 Russcher M, Josephus Jitta N, Kraaijenhagen RJ, Fijnheer R, Pasker-de Jong PC, Gaillard CA: Preemptive dosage reduction of nadroparin in patients with renal failure: a retrospective case series. Clin Kidney J 2013;6:473477.

18 Schmid P, Fischer AG, Wuillemin WA: Lowmolecular-weight heparin in patients with renal insufficiency. Swiss Med Wkly 2009;139: 438-452.

19 Leri F, Voyce SJ, Scialla S, Glavich W, Dzielak E, Smego RA Jr, Guzek J: Enoxaparin dosing in the elderly using adjusted body weight. J Thromb Thrombolysis 2009;28:348-353.
20 Atiq F, van den Bemt PM, Leebeek FW, van Gelder T, Versmissen J: A systematic review on the accumulation of prophylactic dosages of low-molecular-weight heparins (LMWHs) in patients with renal insufficiency. Eur J Clin Pharmacol 2015;71: 921-929.

21 Nagge J, Fernandes O, Huh J: Evaluation of a pharmacokinetic program for adjusting enoxaparin in renal impairment. Am Heart J 2005;149:e21; author reply e23-e24.

22 Kruse MW, Lee JJ: Retrospective evaluation of a pharmacokinetic program for adjusting enoxaparin in renal impairment. Am Heart J 2004; 148:582-589.

23 Montalescot G, Collet JP, Tanguy ML, Ankri A, Payot L, Dumaine R, Choussat R, Beygui F, Gallois V, Thomas D: Anti-Xa activity relates to survival and efficacy in unselected acute coronary syndrome patients treated with enoxaparin. Circulation 2004; 110:392398.

24 Barrett JS, Hainer JW, Kornhauser DM, Gaskill JL, Hua TA, Sprogel P, Johansen K, van Lier JJ, Knebel W, Pieniaszek HJ Jr: Anticoagulant pharmacodynamics of tinzaparin following $175 \mathrm{iu} / \mathrm{kg}$ subcutaneous administra- tion to healthy volunteers. Thromb Res 2001; 101:243-254.

25 Cheer SM, Dunn CJ, Foster R: Tinzaparin sodium: a review of its pharmacology and clinical use in the prophylaxis and treatment of thromboembolic disease. Drugs 2004;64: 1479-1502.

26 Cambus JP, Saivin S, Heilmann JJ, Caplain H, Boneu B, Houin G: The pharmacodynamics of tinzaparin in healthy volunteers. Br J Haematol 2002;116:649-652.

27 Hainer JW, Barrett JS, Assaid CA, Fossler MJ, Cox DS, Leathers T, Leese PT: Dosing in heavy-weight/obese patients with the LMWH, tinzaparin: a pharmacodynamic study. Thromb Haemost 2002;87:817-823.

28 Bounameaux $\mathrm{H}$, de Moerloose P: Is laboratory monitoring of low-molecular-weight heparin therapy necessary? No. J Thromb Haemost 2004;2:551-554.

29 Harenberg J: Is laboratory monitoring of lowmolecular-weight heparin therapy necessary? Yes. J Thromb Haemost 2004;2:547-550.

30 Nagge J, Crowther M, Hirsh J: Is impaired renal function a contraindication to the use of low-molecular-weight heparin? Arch Intern Med 2002;162:2605-2609.
Empirically Reduced Dosages of Tinzaparin in Patients
Nephron 2017;137:113-123 DOI: $10.1159 / 000477474$ 